\title{
Were freak waves involved in the sinking of the Tanker "Prestige"?
}

\author{
A. Lechuga \\ CEDEX Ministry of Fomento, Madrid, Spain
}

Received: 12 July 2006 - Revised: 16 November 2006 - Accepted: 16 November 2006 - Published: 21 November 2006

\begin{abstract}
This paper deals with the possible involvement of freak, rogue or giant waves in the damage suffered by the Tanker Prestige, which eventually led to its sinking. By reason of their very characteristics, giant waves are hard to record. Their more or less sudden appearance makes them fairly elusive objects, except for the consequences they produce. However, some hints with regard to the probability of their occurrence can be derived from considering how close the maritime weather of the area of interest is to the situation which is optimal for their generation.
\end{abstract}

This paper takes into account the wave field in the area and at the time of the Prestige accident and investigates how near or how far the wave field was to the instability conditions that are favourable to the generation of freak waves in the different approximations. This paper explores mostly the modulation instability which is one of the most common mechanisms to produce freak waves: it leads to the decomposition of an initially homogeneous train of Stokes waves firstly into a series of groups of waves, whose envelope then produces the so-called "solitons", that then collapse in the form of a giant wave. This mechanism mainly occurs in deep waters. Zakharov studied it in 1968 and, independently, Benjamin and Feir analysed it in 1967.

This paper proves that the wave field was conspicuously two dimensional with two main wave components travelling in directions almost orthogonal to each other. This means that the wave field was well outside the instability domain. Therefore it is concluded that freak waves were very unlikely generated and it is improbable that they were responsible for the accident.

Correspondence to: A. Lechuga

(antonio.lechuga@cedex.es)

\section{Introduction}

Some people and institutions concerned with the accident of the Tanker Prestige expressed the opinion that perhaps the main damage that eventually led to its sinking could be caused by freak waves. In order to clarify this point we are going to study the possible involvement of such waves in the damage suffered by the Prestige, using observed wave records and theoretical models to describe the sea conditions.

Firstly, it is useful to define what we understand as a freak wave. To this end we will take into account the descriptions appearing in the literature and the opinions shared by many researchers, such as Pelinovsky and Kharif (2000), Peregrine (1979), Osborne et al. (2000), Zakharov et al. (2006), and Dyachencko and Zakharov (2005), amongst many others. The two last named authors, in particular, point out the following characteristics of freak waves:

1. They are essentially non-linear "objects".

2. Freak waves steepness can be so great that they look like a wall of water.

3. They are single events.

4. Before breaking, their crest is three or four (or more) times higher than the crests of the surrounding waves.

5. They are preceded by a kind of hole in the sea.

6. They appear almost instantaneously in an otherwise relatively calm sea.

7. They are short-lived, surviving only for a few minutes.

There are other, more statistical definitions. Some authors, for instance, suggest that freak or rogue waves should be defined as those in which the maximum wave height is 2 to 3 times greater than the significant wave height for a given storm. 


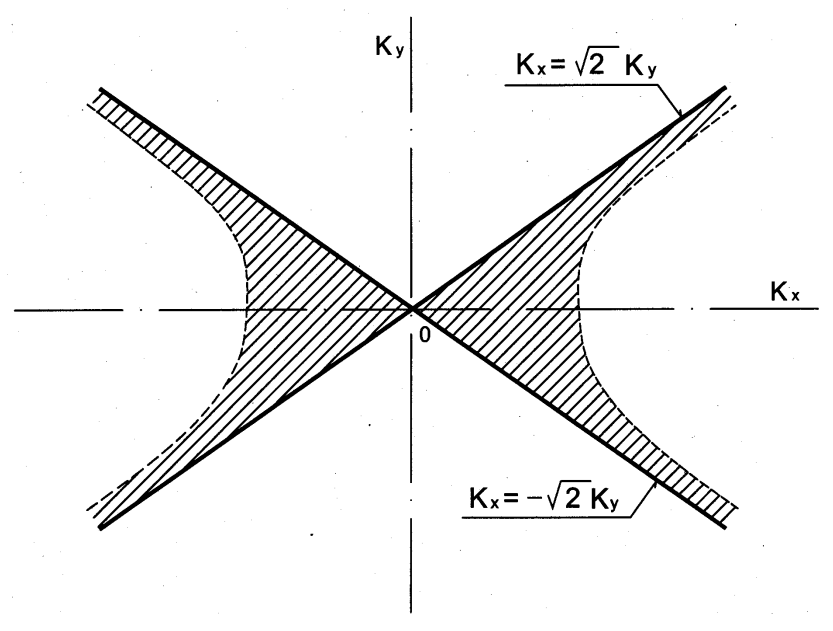

Fig. 1. Instability domain in the two dimensional case.

Various different mechanisms have been put forward as being responsible for the generation of this kind of waves. In the next paragraphs we summarize the most important ones.

One mechanism is the interaction of waves with a strong current. A well-known example of this kind is the occurrence of giant waves with the above-mentioned characteristics in the area of the famous Aghulas current.

A second mechanism is related to the caustic theory in geometrical optics. In short, this means concentration of waves in space and time. As Peregrine (1988) pointed out, the concentration of waves in wave propagation resulting from ray theory is partially eliminated when the nonlinear effects are taken into account. This is not the case, however, if we are faced with geometrical focussing, which has been put forward as a freak wave generating mechanism by Pelinovsky et al. (2005).

A third mechanism is the so-called Stokes waves modulation instability, which has been studied by many authors and is known as the Benjamin-Feir (1967) instability, although Zakharov (1968) arrived independently at the same results. We will look at this mechanism in greater detail in the following section. The instability manifests itself in the modulation of the amplitude of the waves.

There is a fourth generating mechanism, which is related to the frequency modulation of wave packets that propagate at the wave group speed, as was pointed out by Pelinovsky et al. (2005). This mechanism is effective in coastal areas in the form of edge waves.

\section{Modulation instability}

This instability mechanism leads to the decomposition of an initially homogeneous train of Stokes waves, firstly into a series of groups of waves. Recently Zakharov et al. (2006) have called this state as "quasi-solitonic turbulence". After that,

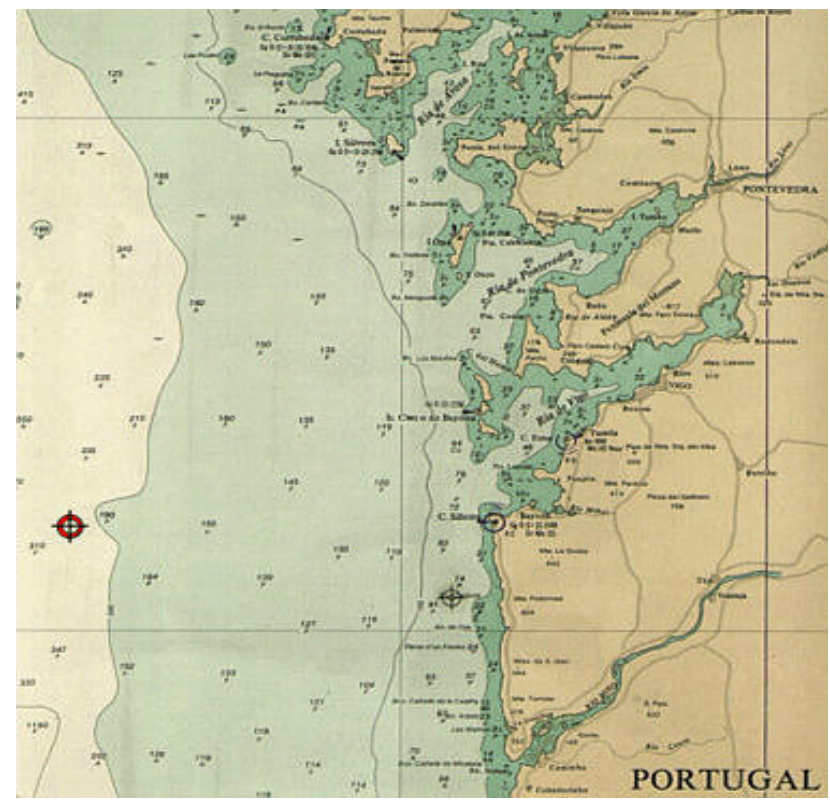

Fig. 2. Location of the Silleiro buoy.

in a process that is still not well understood at present, freak waves appear and collapse. This mechanism mainly occurs in deep waters. Zakharov studied it in 1968 in the framework of the Non-Linear Schrödinger Equation (NLSE), which he deduced. It has therefore been clear since the middle 1960s of the past century that a wave train is unstable for waves of a relatively close frequency, propagating more or less in the same direction. Benjamin and Feir (1967) arrived at the same conclusion using a different approach. The evolution equation in one dimension is:

$i \frac{\partial A}{\partial \tau}-\frac{1}{8} \frac{\omega_{0}}{k^{2}} \frac{\delta^{2} A}{\delta \xi^{2}}-\frac{1}{2} \omega_{0} k^{2}|A|^{2} A=0$

Where $A$ represents the amplitude of the modulated wave, $\tau$ the time and $\xi$ the spatial coordinate. $A$ is a complex variable and, as usual, we move with the reference frame. In the limit of Stokes waves the frequency is:

$\omega=\omega_{0}\left(1+\frac{1}{2} k^{2} a^{2}\right)=\sqrt{g k}\left(1+\frac{1}{2} k^{2} a^{2}\right)$

where $k$ is the wave number of the single Stokes wave.

After Zakharov (1968), to study linear stability we introduce the new variables :

$$
\begin{aligned}
& A(\xi, \tau)=a(\xi, \tau) \exp \left(-i \frac{\omega_{0} k^{2}}{2}\left|a_{0}\right|^{2} \tau\right) \\
& a=a_{0}+b(\xi, \tau) \\
& b(\xi, \tau)=b_{1} \exp (i(K \xi-\Omega \tau))
\end{aligned}
$$

and finally, after introducing these values in Eq. (1) we obtain:

$i b_{\tau}-\frac{\omega_{0}}{8 k^{2}} b_{\xi \xi}-\frac{\omega_{0} k^{2} a_{0}^{2}}{2}(b+\bar{b})=0$ 


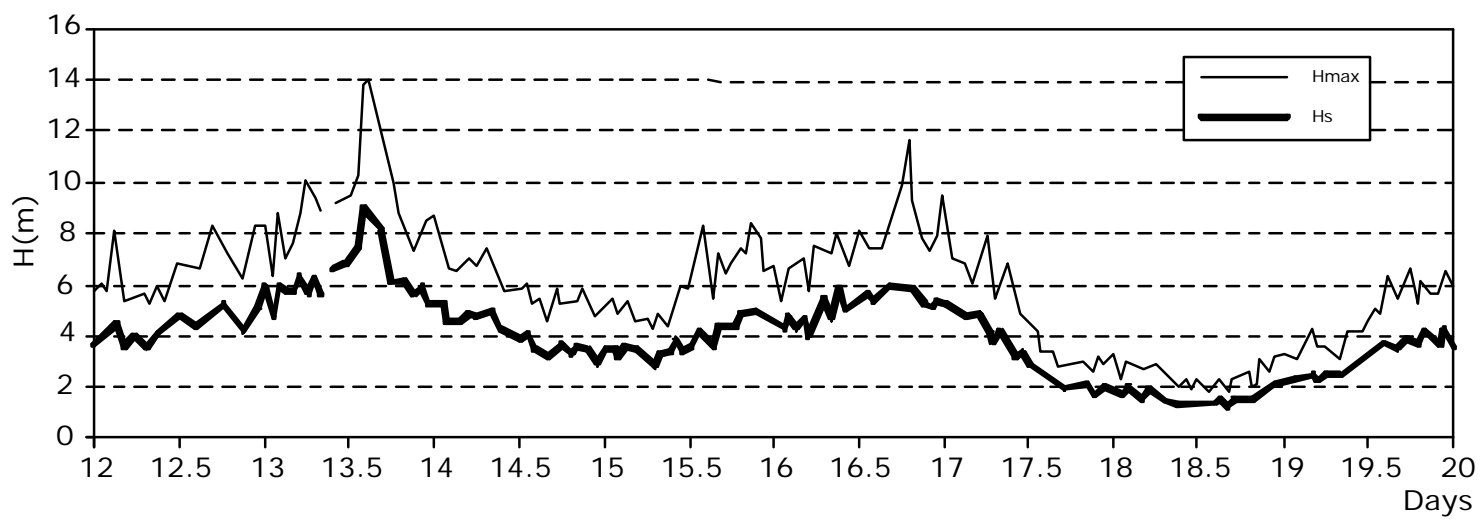

Fig. 3. Records of $H_{S}$ (significant wave height) and $H_{\max }$ (maximum wave height) between 12 and 20 November 2002 . Silleiro buoy.

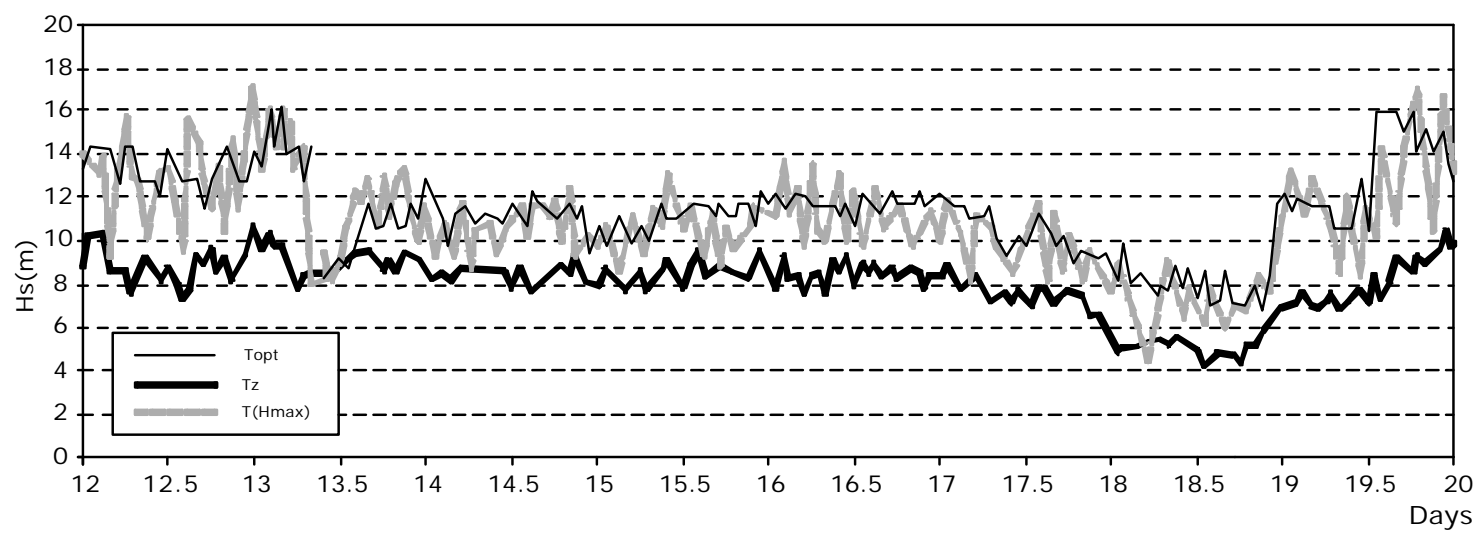

Fig. 4. Records of $T_{z}, T_{\mathrm{opt}}$ and $T\left(H_{\max }\right)$ between 12 and 20 November 2002. Silleiro buoy.

Where, $\bar{b}$ is the complex conjugate of $b$. Equation (4) has been obtained after considering only the linear terms in $b$. Separating real and imaginary parts in Eq. (4) and writing $b=\alpha+i \beta$, we get:

$\alpha\left(\frac{\omega_{0} k^{2} a_{0}^{2}}{2}-\frac{\omega_{0}}{8 k^{2}} K^{2}\right)+\beta \Omega=0$

$\alpha \Omega+-\beta \frac{\omega_{0}}{8 k^{2}} K^{2}=0$

In order to ensure the compatibility of the two equations (5), we obtain:

$$
\begin{aligned}
& \Omega^{2}=-\frac{1}{8} \frac{\omega_{0}}{k^{2}} K^{2}\left(-\frac{1}{8} \frac{\omega_{0}}{k^{2}} K^{2}+2\left(\frac{1}{2} \omega_{0} k^{2}\right) a_{0}^{2}\right) \\
& K^{2} \leq 8 k^{4} a_{0}^{2}
\end{aligned}
$$

where $K$ and $\Omega$ represent the wave number and the frequency of the modulated wave train respectively. The value of $K$ that makes $\Omega$ equal to zero is the instability limit.

Therefore,

$0<\frac{K}{k} \leq 2 \sqrt{2} a_{0} k$
Following Eq. (7), the bigger the steepness of the wave $a_{0} k$, the greater the instability domain is.

When the wave field is characterised by two main directions of wave train propagation, then it can be shown that the instability mechanism is greater, the closer are the directions of the field components.

Zakharov (1968) gave also a two dimensional version of the NLSE that was obtained after simplifying the so-called Zakharov integral equation. This equation is expressed as:

$i \frac{\partial A}{\partial \tau}-\frac{\omega_{0}}{8 k^{2}} \frac{\delta^{2} A}{\delta x^{2}}+\frac{\omega_{0}}{4 k^{2}} \frac{\delta^{2} A}{\delta y^{2}}-\frac{1}{2} \omega_{0} k^{2}|A|^{2} A=0$

Performing the same analysis, Hui and Hamilton (1979) gave solutions to Eq. (8) after converting it in the one-dimension NLSE:

$i \frac{\partial A}{\partial \tau}+\lambda_{1} \frac{\delta^{2} A}{\delta \xi^{2}}-\chi|A|^{2} A=0$

through the coordinate change:

$\xi=x \cos \phi+y \sin \phi$ 


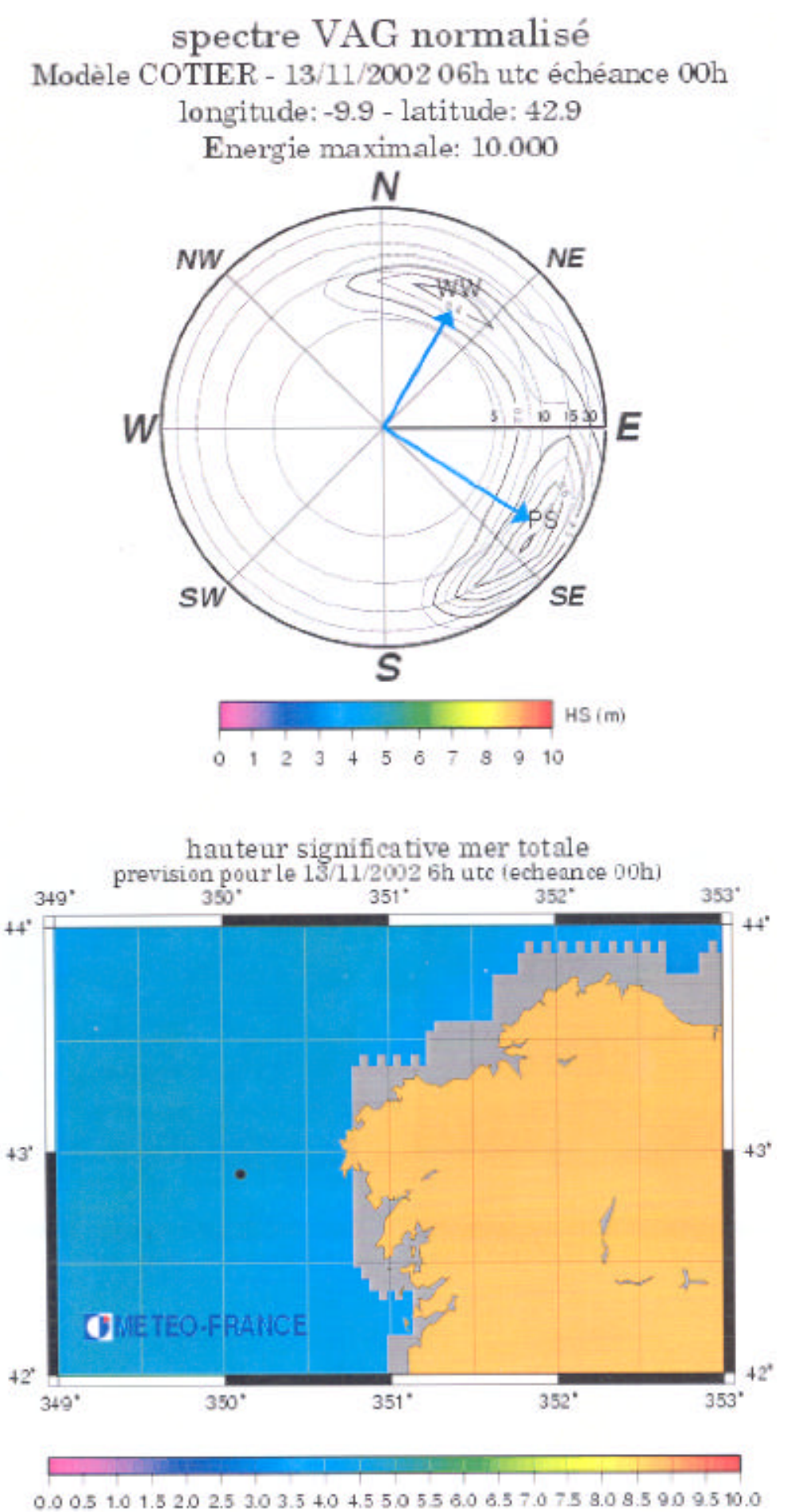

Fig. 5. Directional Spectra on 13 November 2002, 06:00 h, showing two peaks. Méteo France.

In Eq. (9), according to Hui and Hamilton (1979) and Zakharov (1968):

$\lambda_{1}=-\frac{\omega_{0}}{8 k^{2}} \cos ^{2} \phi+\frac{\omega_{0}}{4 k^{2}} \sin ^{2} \phi$
It is easy to prove that $\lambda_{1}$ becomes null when

$|\tan \phi|=2^{-\frac{1}{2}}$ and thus $\phi=35^{\circ}$ or $\phi=145^{\circ}$

Hence, this condition defines the limit of the instability domain. 


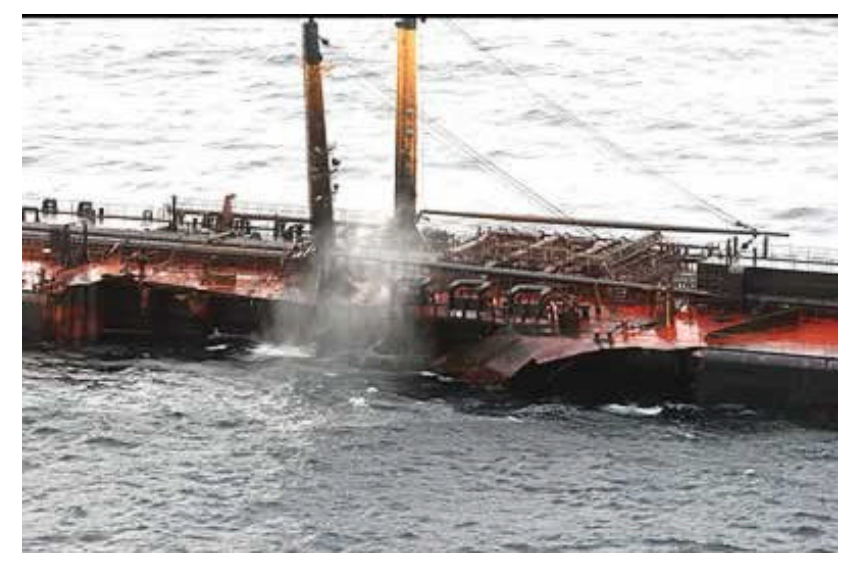

Fig. 6. Initial damage on the Prestige. 13 November 2002.

According to Zakharov (1968), this means that if the two main wave trains propagate along directions forming an angle greater than $35^{\circ}$ (see Fig. 1), they cannot interact with each other making it impossible to produce modulation instability. Enhancements of the NLSE proposed by Dysthe (1979) and by Trulsen and Dysthe (1996) do not change significantly these limits.

\section{Wave characteristics on the day of the accident}

We have already pointed out that giant waves are hard to record. There are a few examples of such records in the technical literature. However, some parameters may be considered to give some hints on the greater or lesser probability of their occurrence, taking into account the closeness of the sea conditions in the area of interest to the state most favourable to generating them.

According to the CEDEX report on maritime weather conditions, and on the data recorded by the Silleiro buoy (see Fig. 2) on 13 November 2002, the significant wave height was $9 \mathrm{~m}$ and the maximum wave height was $14 \mathrm{~m}$ (see Fig. 3). These values are high, but not exceptional. The ratio between the two heights is 1.556 , which is well within the limits of the Rayleigh distribution. From this point of view, the recorded waves are far from being freak waves, if we use the criterion, as most researchers do, of considering freak waves when this ratio is 2 or 3 . In Fig. $4, T_{z}$ is the period of the zero upcrossing waves, $T_{\mathrm{opt}}$ is the period corresponding to the peak of the spectrum (maximum of energy) and $T\left(H_{\max }\right)$ the period corresponding to $H_{\max }$.

The significant wave steepness, $\frac{H_{s}}{L}$, on the same day is 0.062 , where $L$ is the wavelength computed using $T_{Z}$. This steepness expressed in "ak" form (amplitude times wavenumber) would be 0.1948 , which is rather high. Concerning this value, only one thing can be said: bearing in mind Eq. (7), the instability domain is, comparatively, greater than otherwise.

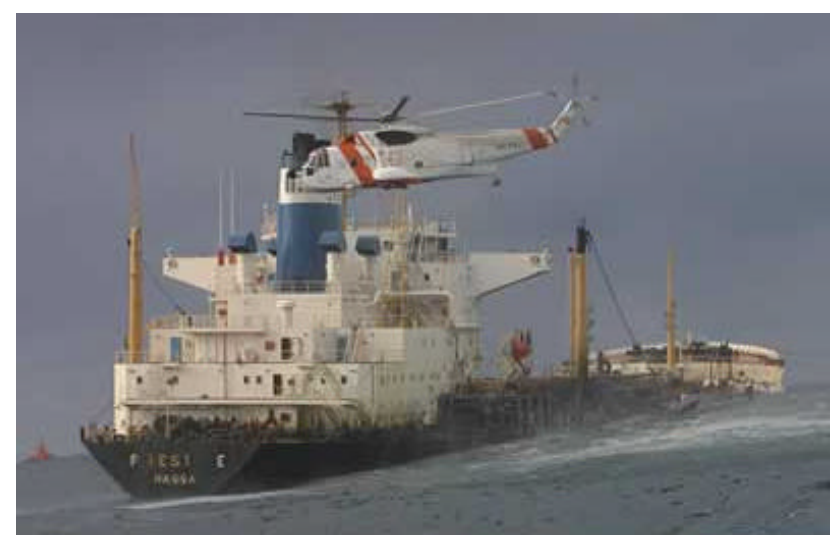

Fig. 7. The Tanker Prestige from the stern.

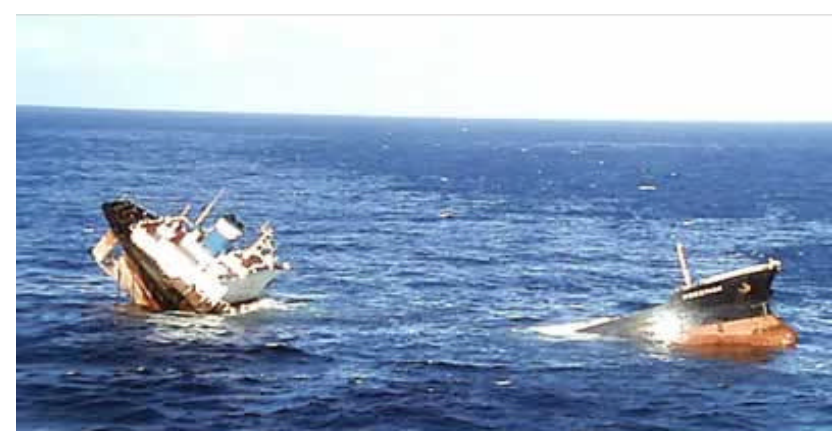

Fig. 8. Sinking of the Tanker Prestige. 20 November 2002.

The ANNEXE 2 (Méteo France) of the Rapport de Situation Météorologique "Prestige" (Bulletins de prévisions météorologiques pour la zone FINISTERRE, les 13 et 14 novembre 2002), shows that directional spectra from 00:00 UTC to 15:00 UTC mark two almost perpendicular components, one being a NW-SE swell and the other a SW-NE wind-sea wave state (see Fig. 5). This created a two-dimensional wave field, with two fairly pointed spectra whose clearest expression occurs at 06:00 UTC. This characteristics of the maritime climate is the most conclusive in reference to the formation of freak waves in our case, because, taking the $\xi$-axis as the direction of the swell (PS in Fig. 5), the other component lies outside the instability zone.

\section{Conclusions}

Of all the wave characteristics in the area on 13 November 2002, the one that comes closest to rogue wave generating mechanism is the steepness, assuming coincident directions of wave trains with close frequencies. The fact that the wave fronts meet at an angle of $90^{\circ}$ means that the situation is "uncomfortable" or even dangerous for navigation, but it would not appear to constitute an optimal situation for the generation of freak waves, due to the previous point. In fact, in 
the frame of the water wave modulation instability, the generation of freak waves is impossible. For our purpose we have considered only the deep water modulation instability mechanism, because the accident took place at approximately $700 \mathrm{~m}$ depth. The enhancements of the NLSE do not change significantly the limit angle $\left(35^{\circ}\right)$. In some formulations, f.i. Trulsen and Dysthe (1996), this angle is even reduced for higher values of wave numbers.

Freak waves are difficult to record and, at the same time, there are many uncertainties and many researches (both, theoretical and experimental) to be carried out upon the subject in the future. This leads us to be particularly cautious when considering them as a possible cause of maritime accidents.

Acknowledgements. The author thanks the comments and suggestions of the Editor S. Tinti and the anonymous reviewer who have contributed significantly to the improvement of the text. Also he thanks the Spanish Dirección General de la Marina Mercante and Méteo France for the use of some pictures and graphs.

Edited by: S. Tinti

Reviewed by: one referee

\section{References}

Benjamin, T. B. and Feir, J. E.: The disintegration of wavetrains in deep water, Part 1, J. Fluid Mech., 27, 417-430, 1967.

Dyachenko, A. I. and Zakharov, V. E.: Modulation Instability of Stokes Wave $\rightarrow$ Freak Wave, J. Exp. Theor. Phys. Lett., 81, 255$259,2005$.
Dysthe, K. B.: Note on a modification to the nonlinear Schrödinger equation for application to deep water, Proc. Roy. Soc. London, A 369, 105-114, 1979.

Hui, W. H. and Hamilton, J.: Exact solutions of a three dimensional nonlinear Schrödinger equation applied for gravity waves, J. Fluid Mech., 93, 117-134, 1979.

Kharif, C., Pelinovsky, E., Talipova, T., and Slunyaev, A.: Focusing of Nonlinear Wave Group in Deep Water, J. Exp. Theor. Phys. Lett., 73, 170-175, 2001.

Osborne, A. R., Onorato, M., and Serio, M.: The Nonlinear Dynamics of Rogue Waves and Holes in Deep-Water Gravity Wave Train, Phys. Lett. A, 275, 386-393, 2000.

Pelinovsky, E. and Kharif, C.: Simplified Model of the Freak Wave Formation from the Random Wave Field, Proc. 15th Int. Workshop on Water Waves and Floating Bodies, Caesaria, Israel, 142145, 2000.

Pelinovsky, E., Lechuga, A., Kurkin, A., Poloukhina, O., and Dubinina, V.: Freak Edge Waves, Proc. of the Fifth International Symposium WAVES 2005, July 2005, Madrid (Spain), CD, 2005.

Peregrine, D. H. and Smith, R.: Nonlinear effects upon waves near caustics, Phil. Trans. Roy. Soc. London, A 292, 341-370, 1979.

Peregrine, D. H., Skyner, D., Stiassnie, M., and Dold, N.: Nonlinear effects on focused water waves, Proc. 21th Int. Conf. on Coastal Engng., 1, 54, 732-742, 1988.

Trulsen, K. and Dysthe, K. B.: A modified nonlinear Schrödinger equation for broader bandwidth gravity waves on deep water, Wave Motion, 24, 281-289, 1996.

Zakharov, V.: Stability of periodic waves of finite amplitude on surface of deep water, J. Appl. Mech. Tech. Phys., 2, 190-194, 1968.

Zakharov, V. E., Dyachenko, A. I., and Prokofiev, A. O.: Freak Waves as Nonlinear Stage of Stokes Wave Modulation Instability, preprint, 1-31, 2006. 